OPEN ACCESS

Edited by:

Jamshed lqbal,

COMSATS University Islamabad,

Pakistan

Reviewed by:

Cláudia Sirlene Oliveira,

Pelé Petit Prince Research Institute,

Brazil

Mohamed A. Abdelgawad,

Al Jouf University,

Saudi Arabia

*Correspondence: Jianmin Chen

chenjianmin1985@sina.com

Specialty section: This article was submitted to Experimental Pharmacology and Drug Discovery,

a section of the journal

Frontiers in Pharmacology

Received: 29 October 2019 Accepted: 27 January 2020

Published: 06 March 2020

Citation:

Chen J, Ye Y, Ran M, Li Q, Ruan Z and Jin N (2020) Inhibition of Tyrosinase by Mercury Chloride: Spectroscopic and

Docking Studies.

Front. Pharmacol. 11:81.

doi: 10.3389/fphar.2020.00081

\section{Inhibition of Tyrosinase by Mercury Chloride: Spectroscopic and Docking Studies}

\author{
Jianmin Chen ${ }^{1,2 *}$, Yaling $Y e^{1}$, Mengnan Ran ${ }^{1}$, Qinglian $L i^{1}$, Zhipeng Ruan ${ }^{1,2}$ \\ and $\mathrm{Nan} \mathrm{Jin}^{1,2}$ \\ 1 School of Pharmacy and Medical Technology, Putian University, Fujian, China, ${ }^{2}$ Key Laboratory of Pharmaceutical Analysis \\ and Laboratory Medicine, Putian University, Fujian Province University, Fujian, China
}

Inorganic mercury compounds have been used in skin-lightening products since ancient times. Although a previous study demonstrated that mercury impeded the transfer of $\mathrm{Cu}^{2+}$ to the apotyrosinase, the effect of mercury on tyrosinase is still unclear. In the present study, the mechanism of mercury chloride $\left(\mathrm{HgCl}_{2}\right)$ induced inactivation of tyrosinase was investigated for the first time. The $I_{50}$ values were 29.97 and $77.93 \mu \mathrm{mol} / \mathrm{L}$ for monophenolase and diphenolase, respectively. A kinetic analysis revealed that $\mathrm{HgCl}_{2}$ inhibited tyrosinase activity in an irreversible non-competitive manner. The strong intrinsic fluorescence quenching suggested that the formation of the $\mathrm{HgCl}_{2}$-tyrosinase complex induced conformational changes of the enzyme, and $\mathrm{HgCl}_{2}$ had only one single binding site or a single class of binding site on tyrosinase. The molecular docking and further experiments demonstrated that $\mathrm{HgCl}_{2}$ bound to the amino residuals (His) in the catalytic center of tyrosinase. To our knowledge, these findings presented in this paper were the first evidence of the direct interactions between $\mathrm{HgCl}_{2}$ and tyrosinase, which provided a deep understanding of the inhibition mechanism of mercury on tyrosinase.

Keywords: mercury chloride, tyrosinase, spectroscopic measurements, molecular docking, inhibition mechanism

\section{INTRODUCTION}

Tyrosinase (EC 1.14.18.1), a type III copper protein, is ubiquitously distributed in bacteria, fungi, plants, insects, crustaceans, and mammals (Yoon et al., 2009). It is able to perform two successive reactions: the hydroxylation of tyrosine to dopa (monophenolase activity) and also the subsequent oxidation of dopa to dopaquinone (diphenolase activity) (Körner and Pawelek, 1982; Tan et al., 2016). Dopaquinone, also known as o-dopaquinone, is of high reactivity and its derivatives spontaneously polymerize to form melanin through a series of chemical reactions (Seo et al., 2003). Consequently, tyrosinase plays a pivotal role in melanin formation, which is an important component responsible for skin pigmentation in humans. Although melanin produced in skin melanocytes provides protection from UV radiation, excessive accumulation of melanin may cause cosmetic flaws such as age spots, freckles, senile lentigines, solar lentigo, and melasma in humans (Germanò et al., 2012; Hridya et al., 2016; Soares et al., 2017).

To decrease the abnormal accumulation of melanin, the use of skin-lightening (also known as skin bleaching and skin whitening) products has been prevalent among women all over the world 
for decades (Olumide et al., 2008). The active ingredients known to be effective for skin lightening include various corticosteroids, hydroquinone, and mercury (Barry et al., 2011). Mercury exists primarily in three forms: inorganic, organic, and metallic (or elemental) (Boyd et al., 2000). Inorganic mercury compounds, including mercuric chloride, mercurous chloride, and mercurous oxide, have been used in skin-lightening products since ancient times (Kibukamusoke et al., 1974). These compounds occur in a mercuric (Hg II) or mercurous (Hg I) form and have toxic effects on humans (Clarkson et al., 2003). It has been discovered that the use of skin-lightening products is one of the causes of mercury toxicity (Agrawal and Mazhar, 2015). More specifically, the use of skin-lightening products containing inorganic mercury may lead to central nervous system, gastrointestinal and renal toxicity (Chan, 2011). For this reason, the sale of skin-lightening products containing inorganic mercury was prohibited in many countries (Al-Saleh, 2016). Despite repeated warnings of mercury poisoning by health authorities and the ban against the sale of skin-lightening products containing inorganic mercury, these products are widely available for sale in pharmacies, beauty aid stores and on the internet (Hamann et al., 2014).

The failure in the prohibition of adding inorganic mercury to skin-lightening products is an interesting question worthy of consideration. We believe that the main reason may be the good performance of inorganic mercury compounds in skinlightening and anti-freckle effects. So now comes the question, why do these compounds exhibit such strong inhibition ability of melanin production. It is well known that mercury can replace the copper needed for tyrosinase activity, thereby deactivating enzymes and producing skin-lightening effects (Lerner, 1952a). Specifically, mercury ions can compete with copper ions for active centers on apotyrosinase to produce an inactive preparation; it is worth noting that once copper becomes attached to the enzyme it is replaced by ion of mercury with difficulty (Lerner, 1952b). That is to say, the previous literature (Lerner, 1952b) has demonstrated that mercury only displayed an effect on apotyrosinase as opposed to tyrosinase. Therefore, the direct interaction between mercury and tyrosinase is still unknown. In this study, we used kinetics measurements, spectral investigation, and molecular docking to study the effects of mercury on tyrosinase and tried to elucidate the intrinsic mechanism of the interaction at the molecular level.

\section{MATERIALS AND METHODS}

\section{Materials}

Tyrosine (98\%), dopa (98\%), and mushroom tyrosinase (1000U/ mg, EC 1.14.18.1) were purchased from Sigma-Aldrich (St. Louis, MO, USA). Mercuric chloride $\left(\mathrm{HgCl}_{2}, 99.5 \%\right)$ and kojic acid (99\%) was obtained from Aladdin Bio-technology (Shanghai, China). Histidine (His), valine (Val), glutamate (Glu), and alanine (Ala) were of analytical grade and bought from Xiya Chemical Industry (Shandong, China). All other chemicals and reagents used were of analytical grade or pharmaceutical grade. A Milli-Q-Plus ultra-pure water system from Millipore (Sartorius 611, Germany) was used throughout the study to obtain water used during the experiments.

\section{The Effects on the Monophenolase Activity of Tyrosinase}

The method was modified based on the previous description (Chen et al., 2016). In brief, the assays were carried out by using 3 $\mathrm{mL}$ of reaction system containing $0.5 \mathrm{mmol} / \mathrm{L}$ substrate (tyrosine) in $50 \mathrm{mmol} / \mathrm{L} \mathrm{Na}_{2} \mathrm{HPO}_{4}-\mathrm{NaH}_{2} \mathrm{PO}_{4}$ buffer solution (PBS, $\mathrm{pH}=6.8$ ) at $305 \mathrm{~K}$. A $100 \mu \mathrm{L}$ aliquot of $\mathrm{HgCl}_{2}$ solutions with various concentrations $(0,1.25,5$ and $10 \mathrm{mmol} / \mathrm{L})$ was added to the reaction system to give concentrations ranged from 0 to $333.33 \mu \mathrm{mol} / \mathrm{L}$. The final concentration of tyrosinase was $33.33 \mathrm{U} / \mathrm{mL}$. The optical densities (OD) at wavelength of $475 \mathrm{~nm}$ were recorded using UV spectrophotometer (UV 2550, SHIMADZU) every $30 \mathrm{~s}$ in the first 2 mins and then every 1 min until 30 mins. Kojic acid was used as a positive control. The appropriate blanks (containing both tyrosine and $\mathrm{HgCl}_{2}$ but without tyrosinase) were tested and subtracted to correct the absorbance.

\section{The Effects on the Diphenolase Activity of Tyrosinase}

The method was performed as previously described (Chen et al., 2017). The assays were carried out by using $3 \mathrm{~mL}$ of reaction system containing $0.5 \mathrm{mmol} / \mathrm{L}$ substrate (dopa) in $50 \mathrm{mmol} / \mathrm{L}$ PBS $(\mathrm{pH}=6.8)$ at $305 \mathrm{~K}$. The final concentration of tyrosinase was $13.33 \mathrm{U} / \mathrm{mL}$. A $100 \mu \mathrm{L}$ aliquot of $\mathrm{HgCl}_{2}$ solutions with various concentrations $(0.15,0.31,0.625,1.25,5,10$, and and $20 \mathrm{mmol} / \mathrm{L}$ ) was added to the reaction system to give concentrations ranged from 10.42 to $666.67 \mu \mathrm{mol} / \mathrm{L}$. The OD values of the reaction system in the presence of $\mathrm{HgCl}_{2}$ solutions $\left(\mathrm{A}_{1}\right)$ were recorded at the wavelength of $475 \mathrm{~nm}$ using the UV spectrophotometer. The OD values of the reaction system in the absence of $\mathrm{HgCl}_{2}$ and tyrosinase were used as a positive $\left(\mathrm{A}_{2}\right)$ and a negative $\left(A_{3}\right)$ control, respectively. Relative enzymatic activity $(\%)=\left(A_{1}-A_{3}\right) / A_{2} \times 100 \%$. The experiment was repeated three times. The $\mathrm{IC}_{50}$ value was calculated as the concentration (leading to achieve 50\% inhibition) according to the equation, which is obtained by curve fitting of the enzyme activity versus the concentrations of inhibitor.

\section{Immobilized Tyrosinase for Analysis of Inhibitory Mechanism}

Experiments were conducted to confirm the inhibitory mechanism of $\mathrm{HgCl}_{2}$ on tyrosinase furtherly by using immobilized tyrosinase. In brief, we added $200 \mu \mathrm{L}$ of diluted tyrosinase $(2500 \mathrm{U} / \mathrm{mL})$ to each well of a 24 -well polystyrene plate and then covered the plate and incubated it at $4^{\circ} \mathrm{C}$ overnight to produce immobilized tyrosinase. The plate was brought to room temperature, and we thoroughly decanted the solution from wells and washed the wells two times with PBS to remove the unfixed enzyme. We added $3 \mathrm{~mL}$ of inhibitor solution to each well and incubated them at room temperature for 30 mins to assure the fully reaction between the inhibitor and 
the enzyme, discarding the liquid following by washing the wells two times with PBS to remove the excess inhibitor. Then, $3 \mathrm{~mL}$ of PBS was added to each well, and they stood for 10 mins to make the dissociation of inhibitor-tyrosinase complex. WE thoroughly decanted the solution from wells to remove the dissociated inhibitor (the procedure was repeated three times). Finally, $3 \mathrm{~mL}$ of substrate (dopa) was added to each well and react at room temperature for 30 mins to test the residual activity of tyrosinase. The tested samples included PBS (negative control), 95\% ethanol (positive control of irreversible inhibitor), kojic acid (positive control of reversible inhibitor), and $\mathrm{HgCl}_{2}$, and each sample was tested three times. The concentration of Kojic acid and $\mathrm{HgCl}_{2}$ was both $666.67 \mu \mathrm{mol} / \mathrm{L}$.

\section{Kinetic Analysis for Non-Competitive Inhibition}

The non-competitive inhibition type of tyrosinase was assayed by a Lineweaver-Burk plot, which can be described as the following equation:

$$
\frac{1}{\mathrm{~V}}=\frac{K_{m}}{\mathrm{~V}_{\max }}\left(1+\frac{[\mathrm{I}]}{K_{i}}\right) \frac{1}{[\mathrm{~S}]}+\frac{1+\frac{[\mathrm{I}]}{K_{i}}}{\mathrm{~V}_{\max }}
$$

and a secondary plot is presented below

$$
\text { In . }=\frac{[\mathrm{I}]}{\mathrm{K}_{\mathrm{i}} \mathrm{V}_{\max }}+\frac{1}{\mathrm{~V}_{\max }}
$$

where $v$ is velocity of the enzyme reaction calculated by a change in absorbance at the wavelength of $475 \mathrm{~nm}$ per minute in the absence and presence of $\mathrm{HgCl}_{2} . K_{\mathrm{m}}$ and $K_{\mathrm{i}}$ are the MichaelisMenten constant and inhibition constant, respectively. The values of $K_{\mathrm{m}}$ can be calculated from the equation (1). [I] and $[S]$ are the concentrations of inhibitor and substrate, respectively. In. is the intercept value of the Lineweaver-Burk curve. $K_{\mathrm{i}}$ should be calculated by the secondary plot of In. vs. [I].

\section{Intrinsic Fluorescence Quenching}

The fluorescence emission spectra were obtained using Spectrofluorometer FS5 (Edinburgh Instruments, England) equipped with a $150 \mathrm{~W}$ xenon lamp and a thermostat bath. We added a $2.0 \mathrm{~mL}$ aliquot of tyrosinase solution $(400 \mathrm{U} / \mathrm{mL})$ to the quartz cuvette ( $1 \mathrm{~cm}$ path length) and gradually added $2 \mathrm{mmol} / \mathrm{L}$ solution of $\mathrm{HgCl}_{2}$ to give concentrations ranged from 0 to 400 $\mu \mathrm{mol} / \mathrm{L}$. After five-minute incubation periods, fluorescence in the range of $290-500 \mathrm{~nm}$ was measured while the excitation wavelength was fixed at $280 \mathrm{~nm}$. All the fluorescence data collected from these experiments were corrected for absorption of excitation light and re-absorption of emitted light according to the following equation: (Wang et al., 2015)

$$
F_{c}=F_{m} e^{\left(A_{1}+A_{2}\right) / 2}
$$

where $F_{\mathrm{c}}$ and $F_{\mathrm{m}}$ represent the corrected and measured fluorescence, respectively. $A_{1}$ and $A_{2}$ are the absorbance of $\mathrm{HgCl}_{2}$ at excitation and emission wavelengths, respectively.

\section{Molecular Docking Study}

Molecular docking study was performed by using AutoDock 4.2.6. The X-ray crystal structure of Agaricus bisporus tyrosinase (PDB ID: 2Y9W) and the 3D structure of $\mathrm{HgCl}_{2}$ were both retrieved from the RCSB Protein Data Bank (Ismaya et al., 2011). All input files were prepared using an AutoDockTools (ADT) 1.5.4 package, and a charge of +2 was assigned for copper ions. In order to carry out the docking simulation, a $60 \AA$ × $60 \AA$ × $60 \AA$ -point grid with a spacing of $0.375 \AA$ centered at $4.827,28.489$, $92.878 \AA$ was defined, which fully enclosed the catalytic center of tyrosinase. The AutoGrid program was used to construct the grid maps for energy scoring. The three-dimensional location and orientation of the $\mathrm{HgCl}_{2}$ was explored using the Lamarckian genetic algorithm (LGA). The docking results generated 100 conformations of $\mathrm{HgCl}_{2}$, which were grouped into clusters by a root mean square (RMS) deviation tolerance of 2.0. The lowest energy (optimal conformation) and the largest number cluster (suboptimal conformation) were chosen for further analyses with the PyMOL molecular graphics system.

\section{The Interactions Between $\mathrm{HgCl}_{2}$ and Amino Acid}

According to the results of docking study, we furtherly checked the interactions between $\mathrm{HgCl}_{2}$ and amino acid. We assumed that if $\mathrm{HgCl}_{2}$ indeed binds to the amino acid residues of the enzyme, such as His, Val, Glu, and Ala, premixing the amino acid with $\mathrm{HgCl}_{2}$ would affect the inhibitory effects. Thus, the monophenolase and diphenolase activity of tyrosinase both were determined by adding the mixture of $\mathrm{HgCl}_{2}$ and amino acid. The mixture was prepared just before use by adding an aliquot of $200 \mu \mathrm{L} \mathrm{HgCl}_{2}$ solution to the equal volume of amino acid solution, and which was allowed to react for 10 mins at room temperature. Furtherly, to prove the interaction between His and $\mathrm{HgCl}_{2}$, the UV-Vis spectra of His, $\mathrm{HgCl}_{2}$, and the mixture of both were measured. In brief, 5 and $10 \mathrm{mmol} / \mathrm{L}$ solutions of $\mathrm{His}, \mathrm{HgCl}_{2}$ were prepared beforehand, and the mixture was produced by mixing the His and $\mathrm{HgCl}_{2}$ solutions $(10 \mathrm{mmol} / \mathrm{L})$ in the volume ratio of $1: 1$. The spectra of the samples at the wavelengths between 200 and $800 \mathrm{~nm}$ were scanned by using UV spectrophotometer (UV 2550, SHIMADZU).The experiment was repeated three times.

\section{Statistical Analysis}

Each data point of the experimental results, including tyrosinase activity assay, kinetic analysis for non-competitive type inhibition, fluorescence quenching test, and interactions between amino acid and $\mathrm{HgCl}_{2}$, was repeated at least three times. The data are presented as the mean \pm SD (standard deviation). The statistical significance was determined at the level of $\mathrm{P}$-value $(<0.05)$ by one-way analysis of variance. 

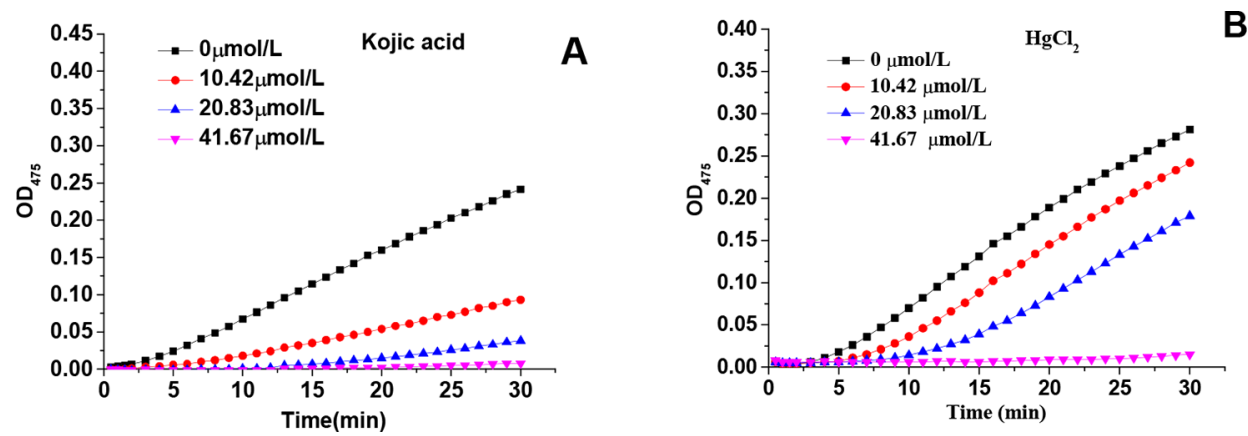

B

FIGURE 1 | Effects of various concentrations of kojic acid and $\mathrm{HgCl}_{2}$ on monophenolase: progression of tyrosine oxidation by tyrosinase in the presence of various concentrations of kojic acid (A) and $\mathrm{HgCl}_{2}$ (B).

\section{RESULTS AND DISCUSSION}

\section{Effects of $\mathrm{HgCl}_{2}$ on the Monophenolase Activity of Tyrosinase}

Figures 1A, B showed the kinetic progression of tyrosine oxidation by tyrosinase in the presence of various concentrations of kojic acid and $\mathrm{HgCl}_{2}$, respectively. With the increase of kojic acid concentration, the lag time was prolonged from $1.44 \mathrm{~min}$ to approximately $10.33 \mathrm{~min}$. Similarly, $\mathrm{HgCl}_{2}$ also exhibited a marked inhibitory effect with significant prolongation of the lag period from 2.00 to 13.4 mins. The inhibitory effects of $\mathrm{HgCl}_{2}$ on monophenolase were activity dependent on the concentrations because the steady rate (the slope of linear part of the kinetic equation) decreased with the increasing concentration of the inhibitors (Park et al., 2005). The $\mathrm{IC}_{50}$ values of kojic acid and $\mathrm{HgCl}_{2}$ were 13.10 and $29.97 \mu \mathrm{mol} / \mathrm{L}$, respectively, suggesting that $\mathrm{HgCl}_{2}$ had less inhibitory capability than kojic acid on monophenolase activity.

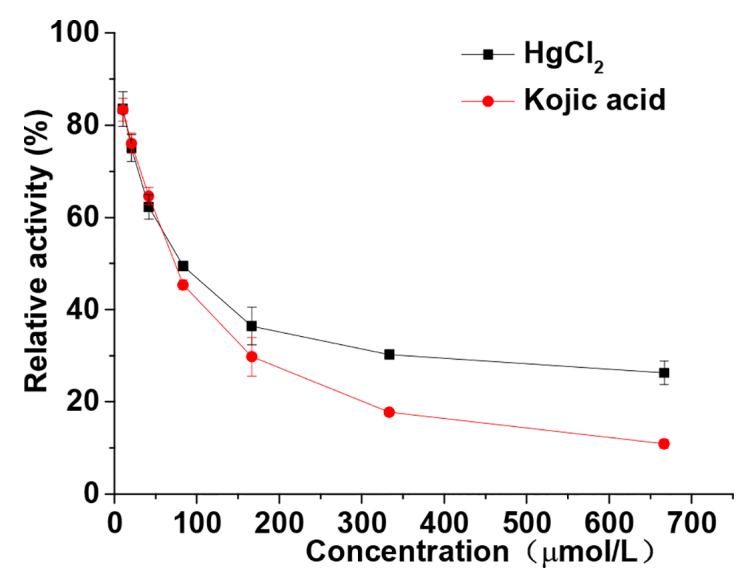

FIGURE 2 | Relative activity of tyrosinase through diphenolase reactions with dopa in the presence of various concentrations (from 10.42-666.67 $\mu \mathrm{mol} / \mathrm{L}$ ) of $\mathrm{HgCl}_{2}$ and kojic acid. The final concentrations of dopa were $0.5 \mathrm{mmol} / \mathrm{L}$. Data are presented as mean \pm standard deviation $(n=3)$.

\section{Effect of $\mathrm{HgCl}_{2}$ on the Diphenolase Activity of Tyrosinase}

Both kojic acid and $\mathrm{HgCl}_{2}$ significantly inhibited the diphenolase activity in a dose-dependent manner, as shown in Figure 2. With an increase in the concentration of kojic acid and $\mathrm{HgCl}_{2}$, the diphenolase activities decreased rapidly with $\mathrm{IC}_{50}$ values of 73.05 and $77.93 \mu \mathrm{mol} / \mathrm{L}$, respectively. $\mathrm{HgCl}_{2}$ exhibited significant inhibitory effect on the monophenolase activity also showed the same inhibitory effect on diphenolase activity, indicating it could inhibit the melanin synthesis in the early enzymatic stages. It is well-known that mercury replaces the copper required for tyrosinase activity, but once copper becomes attached to the enzyme it is replaced by ions of mercury with difficulty (Lerner, 1952b). That is to say, mercury was commonly considered to inhibit the melanin production by preventing the formation of the activated tyrosinase via replacement of copper ions. However, in this study, we found that mercury could inhibit both the monophenolase and diphenolase activity of tyrosinase by binding to the enzyme directly instead of replacing the copper ions required for tyrosinase.

\section{Inhibition Mechanism of $\mathrm{HgCl}_{2}$ on Tyrosinase}

The relationship between enzyme activity (expressed as the rate of the oxidation reaction) and its concentration $(6.67,13.33,20$, 26.67 , and $33.33 \mathrm{U} / \mathrm{mL}$ ) in the presence of different concentrations of $\mathrm{HgCl}_{2}(0,4.17,16.67,33.33$, and $66.67 \mu \mathrm{mol} / \mathrm{L})$ was investigated. A series of parallel (or roughly parallel) lines with the same slope and different abscissa intercepts (Figure 3A) were observed from the plots of tyrosinase activity versus the concentrations of enzyme in the presence of various concentrations of $\mathrm{HgCl}_{2}$, suggesting the inhibition of $\mathrm{HgCl}_{2}$ on tyrosinase was irreversible (Qiu et al., 2005). The increase of abscissa intercept indicated that the number of effective enzyme molecules decreased due to the irreversible inhibition.

In order to prove the irreversible inhibition furtherly, immobilized tyrosinase was used to study the effects of $\mathrm{HgCl}_{2}$ on tyrosinase, the results of which were shown in Figure 3B. As a negative control, the activity of tyrosinase treated by PBS was 


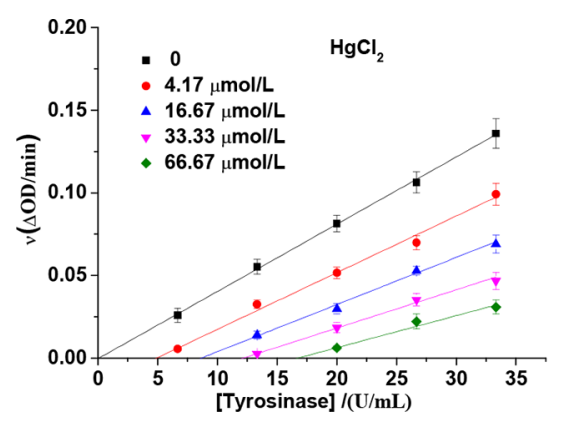

A

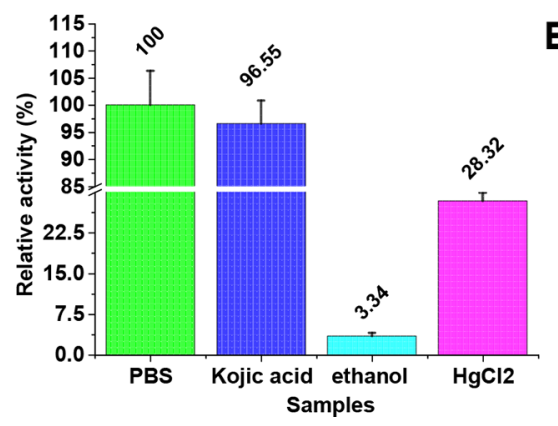

B

FIGURE 3 | Inhibition mechanism of $\mathrm{HgCl}_{2}$ on tyrosinase. (A) An irreversible inhibition of the enzyme on a kinetic basis: the concentrations of $\mathrm{HgCl}_{2}$ were $0,4.17$, 16.67, 33.33, and $66.67 \mu \mathrm{mol} / \mathrm{L}$ for curves, and the concentrations of tyrosinase were 6.67, 13.33, 20, 26.67, and 33.33 U/mL. (B) Immobilized tyrosinase for analysis of inhibitory mechanism: As a negative control, the activity of tyrosinase with PBS treatment was defined as $100 \%$, and the concentration of kojic acid and $\mathrm{HgCl}_{2}$ were both $666.67 \mu \mathrm{mol} / \mathrm{L}$. The experiment was repeated three times $(n=3)$.

defined as $100 \%$. The activity of tyrosinase treated by kojic acid returned to $96.55 \%$ because of the reversible inhibition, whilst treated by $95 \%$ ethanol only returned to $3.34 \%$ because of the irreversible inhibition. The activity of tyrosinase treated by $\mathrm{HgCl}_{2}$ returned to $28.32 \%$, indicating $\mathrm{HgCl}_{2}$ indeed inhibits the tyrosinase in an irreversible way compared to kojic acid. However, the enzyme activity is much higher than the one treated by $95 \%$ ethanol, which may be due to the fact that the amount of the inhibitor $\left(\mathrm{HgCl}_{2}\right)$ is not enough to inactivate the whole enzyme molecules coated on the plate.

\section{Inhibition Type of $\mathrm{HgCl}_{2}$ on the Tyrosinase}

The inhibitory effect of $\mathrm{HgCl}_{2}$ on the diphenolase activity was studied by the Lineweaver-Burk double reciprocal plots. As shown in Figure $\mathbf{4 A}$, the horizontal axis intercept $\left(-1 / K_{\mathrm{m}}\right)$ keeps the value unchanged and the vertical axis intercept $\left(\frac{1+\frac{[I]}{K_{i}}}{V_{\max }}\right)$ increases with the increasing inhibitor concentration, indicating that $\mathrm{HgCl}_{2}$ induced a non-competitive inhibition (Chen et al., 2005). Therefore, $\mathrm{HgCl}_{2}$ could bind both free enzyme and the enzyme-substrate complex with the same inhibition constants $\left(K_{\mathrm{i}}\right)$. The value of $K_{\mathrm{i}}(29.41 \mu \mathrm{mol} / \mathrm{L})$ was obtained from a plot of the vertical axis intercept (intercept of Lineweaver-Burk double reciprocal plots) versus the inhibitor concentration (Mu et al., 2013), as shown in Figure 4B.

\section{Effects of $\mathrm{HgCl}_{2}$ on Tyrosinase Conformational Change}

From the result of the intrinsic fluorescence spectra, we observed that $\mathrm{HgCl}_{2}$ bound to the tyrosinase resulted in conformational changes of tyrosinase: the spectra were gradually decreased in a dose-dependent manner (Figures 5A, B). The concentrations of $\mathrm{HgCl}_{2}$ for the curves (a-f) were $0,95.24,181.82,260.87,333.33$, and $400.00 \mu \mathrm{mol} / \mathrm{L}$, respectively. Although the decrease in the fluorescence intensity were caused by quenching, there were no significant wavelength shift with the accumulation of $\mathrm{HgCl}_{2}$, indicating that tyrosinase does not undergo conspicuous overall structural changes. Moreover, the fluorescence intensity decreased with the increasing concentration of the inhibitor because of the tryptophan-masking properties of $\mathrm{HgCl}_{2}$, which implied that the tyrosinase became disagglomerated, and its structure was loosened (Donghyun et al., 2006).

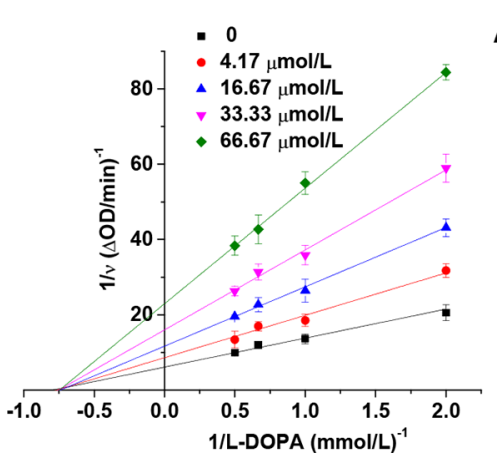

A

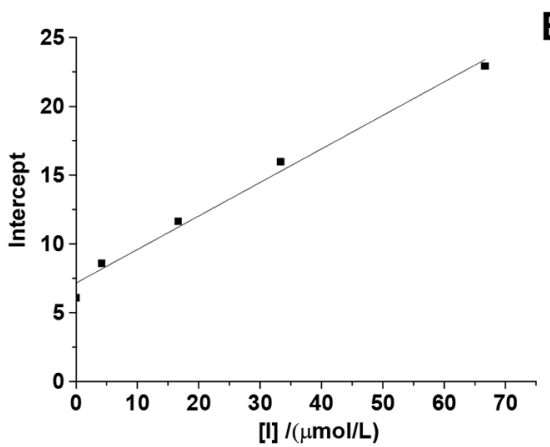

FIGURE 4 | Lineweaver-Burk plot of mushroom tyrosinase in the presence of $\mathrm{HgCl}_{2}: \mathrm{c}(\mathrm{tyrosinase})=13.33 \mathrm{U} / \mathrm{mL}, \mathrm{c}\left(\mathrm{HgCl}_{2}\right)=0,4.17,16.67,33.33$, and $66.67 \mu \mathrm{mol} / \mathrm{L}$ for curves, respectively. The experiment was repeated three times $(n=3)$. 


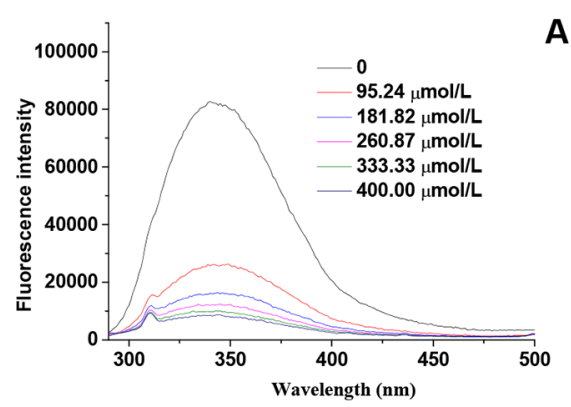

A

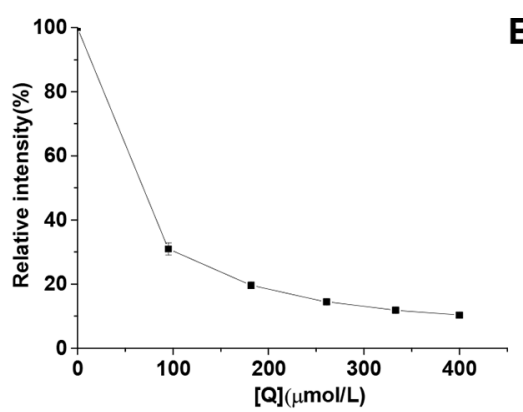

B
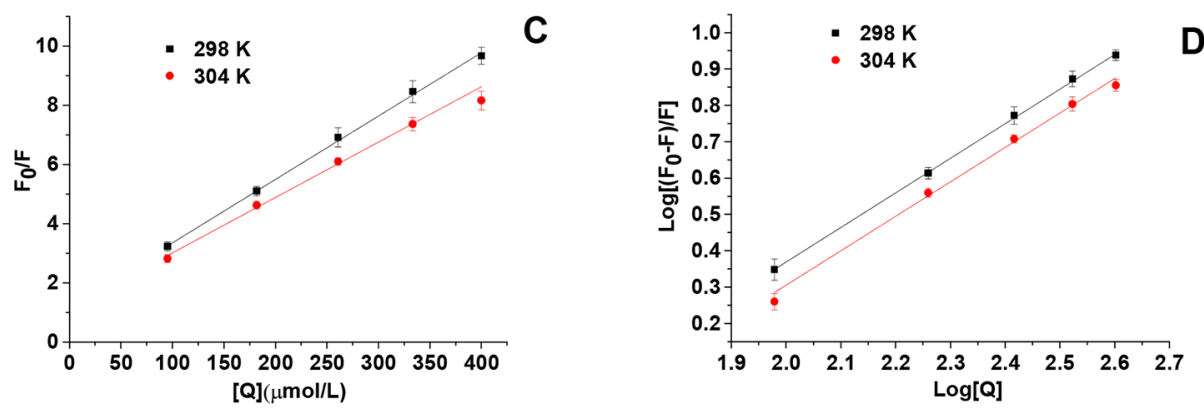

FIGURE 5 | (A) Intrinsic fluorescence spectra of tyrosinase in the presence of $\mathrm{HgCl}_{2}$ at various concentrations, which were $0,95.24,181.82,260.87,333.33$, and $400.00 \mu \mathrm{mol} / \mathrm{L}$ for curves, respectively. (B) Relative intensity (\%) of tyrosinase at various concentrations of $\mathrm{HgCl}_{2}$ (298K). (C) The Stern-Volmer plots for the fluorescence quenching of tyrosinase at various temperatures (298 and $304 \mathrm{~K})$. (D) Plots of log $\left[\left(\mathrm{F}_{0}-\mathrm{F}\right) / \mathrm{F}\right]$ against log $[\mathrm{Q}]$ at various temperatures (298 and $\left.304 \mathrm{~K}\right)$. $\mathrm{F}_{0}$ and $\mathrm{F}$ are the fluorescence intensities of tyrosinase in the absence and presence of $\mathrm{HgCl}_{2}$, and $[\mathrm{Q}]$ represents the concentration of $\mathrm{HgCl}_{2}$. The experiment was repeated three times $(n=3)$.

To identify the interaction mechanism of $\mathrm{HgCl}_{2}$ on tyrosinase, the data of fluorescence quenching were presented in the form of a Stern-Volmer plot, using the following equation:

$$
F_{0} / F=1+K_{\mathrm{q}} \tau_{0}[\mathrm{Q}]=1+K_{\mathrm{sv}}[\mathrm{Q}]
$$

where, $F_{0}$ and $F$ represent the steady-state fluorescence intensities in the absence and presence of $\mathrm{HgCl}_{2}$ (quencher), respectively, [Q] denotes the concentration of $\mathrm{HgCl}_{2}, K_{\mathrm{q}}$ is the quenching rate constant of the biomolecule $\left(K_{\mathrm{q}}=K_{\mathrm{sv}} / \tau_{0}\right), K_{\mathrm{sv}}$ is the Stern-Volmer dynamic quenching constant, and $\tau_{0}$ is the average lifetime of the fluorophore in the absence of quencher $\left(\tau_{0}=10^{-8} \mathrm{~s}\right)$. The value of $K_{\mathrm{sv}}$ determined by the linear regression plot of $\mathrm{F}_{0} / \mathrm{F}$ vs. $[\mathrm{Q}]$ at 298 and $304 \mathrm{~K}$ were $(2.14 \pm 0.05) \times 10^{4}$ and $1.83 \pm 0.03 \times 10^{4} \mathrm{~L} / \mathrm{mol}$, respectively. The plot (Figure 5C) showed a good linear relationship, indicating that a single type of quenching process (static or dynamic quenching) occurred during the formation of $\mathrm{HgCl}_{2}$-tyrosinase complex. $K_{\mathrm{sv}}$ values decreased gradually with the increase of the temperatures, which manifested that the fluorescence quenching of tyrosinase by $\mathrm{HgCl}_{2}$ was a static quenching process ( $\mathrm{Lin}$ et al., 2018). The corresponding $K_{\mathrm{q}}$ values of $(2.14 \pm 0.05) \times 10^{12}$ and $(1.83 \pm 0.03) \times 10^{12} \mathrm{~L} / \mathrm{mol} / \mathrm{s}$ at 298 and $304 \mathrm{~K}$ were much higher than the maximum scattering collision quenching constant $\left(2.0 \times 10^{10} \mathrm{~L} / \mathrm{mol} / \mathrm{s}\right)$ (Abouzied and Alshihi, 2008), suggesting that static quenching is dominant in the $\mathrm{HgCl}_{2}$-tyrosinase interaction (Wang et al., 2014).

For static quenching interactions, if there are similar and independent sites in biological molecules, the apparent binding constants $\left(K_{\mathrm{a}}\right)$ and the number of binding sites (n) can be obtained from the following equation:

$$
\log \frac{F_{0}-F}{F}=\log K_{\mathrm{a}}+\mathrm{n} \log [\mathrm{Q}]
$$

According to the intercept and slope value of the regression curve (Figure 5D), the values of $K_{\mathrm{a}}$ and $\mathrm{n}$ for metal-tyrosinase interaction were calculated based on equation (5). The $K_{\mathrm{a}}$ value of $(2.87 \pm 0.02) \times 10^{4} \mathrm{~L} / \mathrm{mol}$ achieved the order of magnitude of $10^{4} \mathrm{~L} / \mathrm{mol}$, indicating the moderate binding of $\mathrm{HgCl}_{2}$ to tyrosinase (Fan et al., 2017). The results of the section (INHIBITION MECHANISM OF $\mathrm{HGCL}_{2}$ ON TYROSINASE) showed that $\mathrm{HgCl}_{2}$ had a significant inhibitory effect on tyrosinase that was irreversible. We suggested that $\mathrm{HgCl}_{2}$ might be irreversibly coordinated with the amino acid residues of tyrosinase to form the $\mathrm{HgCl}_{2}$-tyrosinase complexes, resulting in the conformation change of the catalytic center of tyrosinase. Furthermore, the $\mathrm{n}$ values $(0.96 \pm 0.01)$ were close to one, suggesting that there was a single binding site or a single class of binding sites in tyrosinase for $\mathrm{HgCl}_{2}$. 

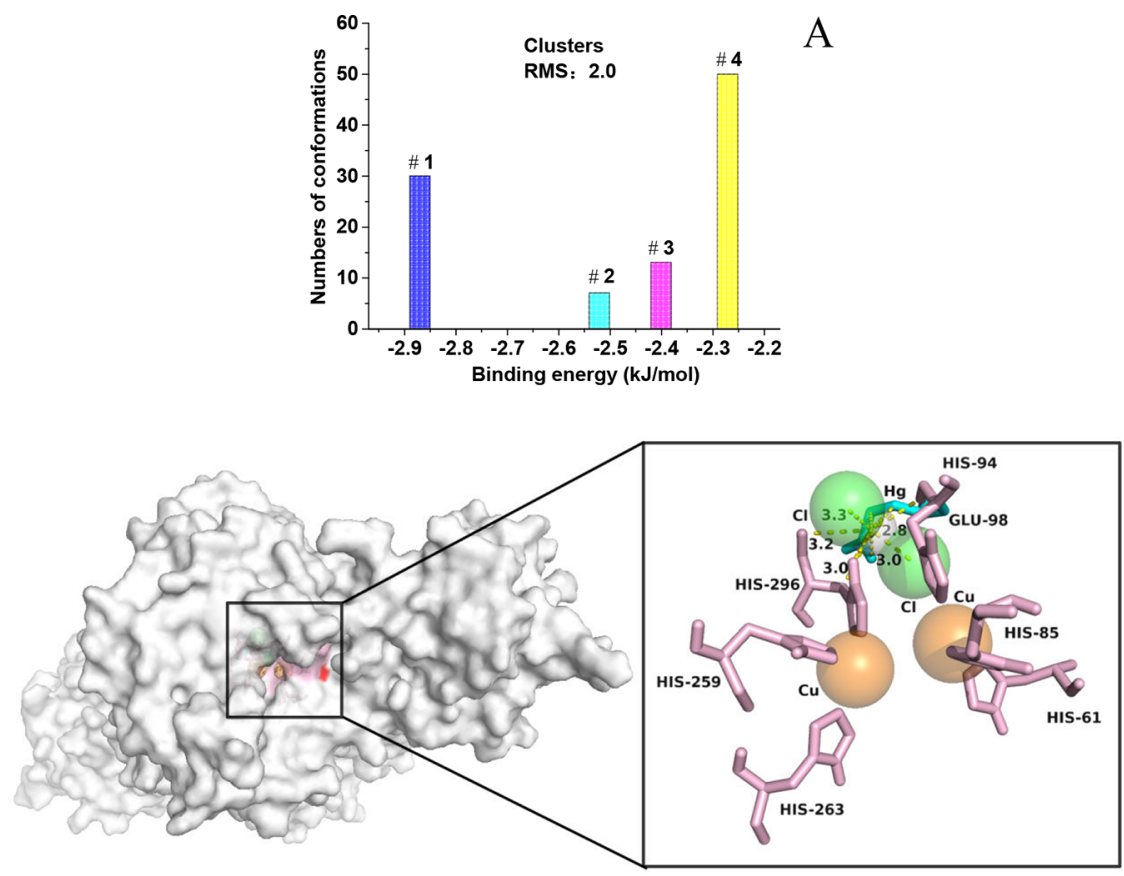

B

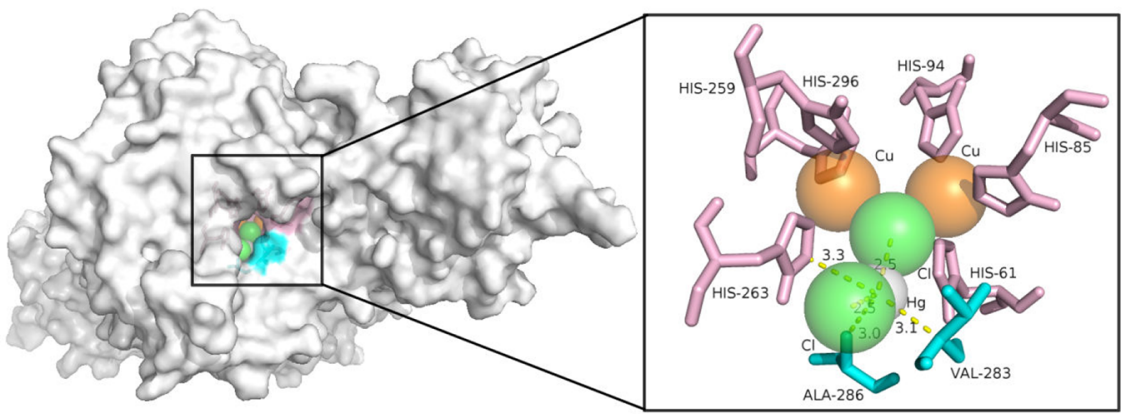

$\mathrm{C}$

FIGURE 6 | (A) Group the conformations into clusters at the specified RMS deviation tolerance of 2.0; (B) details of the optimal conformation; (C) details of the suboptimal conformation.

\section{Docked Conformations}

Molecular docking was furtherly performed to explore the interaction between $\mathrm{HgCl}_{2}$ and tyrosinase. We cluster all the conformations (100) with ADT by a root mean square (RMS) deviation tolerance of 2.0, resulting in four clusters, as shown in Figure 6A. The $X$ position of each bar is plotted by the energy of the lowest energy conformation in the cluster, and the height of the bar represents the number of docked conformations in each cluster. The best docking result (optimal conformation) can be recognized as the lowest energy conformation, and can also be selected according to the RMS deviation from the reference structure (usually the crystallographic bonding mode) (Morris et al., 2008). Thus, a lowest energy $(-2.87 \mathrm{~kJ} / \mathrm{mol})$ conformation in the first cluster is recognized as the optimal conformation, as shown in Figure 6B. A lowest energy $(-2.27 \mathrm{~kJ} / \mathrm{mol})$ conformation in the fourth cluster was recognized as the suboptimal conformation because the conformation-like one with the ligand (tropolone) binds to the catalytic center of tyrosinase (Ismaya
TABLE 1 | Information between the amino acid residues and $\mathrm{HgCl}_{2}$.

\begin{tabular}{|c|c|c|c|c|}
\hline Conformations & $\begin{array}{l}\text { Binding energy } \\
(\mathrm{kJ} / \mathrm{mol})\end{array}$ & Residue & $\begin{array}{c}\text { Atom of } \\
\text { the residue }\end{array}$ & Distance $(\AA ̊)$ \\
\hline \multirow[t]{9}{*}{ Optimal } & -2.87 & His 94 & O & 2.8 \\
\hline & & His 296 & O & 3.2 \\
\hline & & His 296 & $\mathrm{CB}$ & 3.0 \\
\hline & & His 296 & $\mathrm{HD}$ & 3.0 \\
\hline & & Glu 98 & OE & 2.7 \\
\hline & & Glu 98 & ND & 3.6 \\
\hline & & Glu 98 & $C D$ & 3.3 \\
\hline & & Glu 98 & CG & 3.3 \\
\hline & & Glu 98 & CB & 2.8 \\
\hline \multirow[t]{5}{*}{ Suboptimal } & -2.27 & His 263 & CE & 3.3 \\
\hline & & His 263 & ND & 3.5 \\
\hline & & His 263 & $\mathrm{NE}$ & 3.9 \\
\hline & & Ala 286 & $\mathrm{CB}$ & 3.0 \\
\hline & & Val 283 & CA & 3.1 \\
\hline
\end{tabular}

${ }^{a}$ The first character of the atom name consists of the chemical symbol for the atom type. All the atom names beginning with "C" are carbon atoms; " $\mathrm{N}$ " indicates a nitrogen and "O" indicates oxygen. The next character is the remoteness indicator code, which is transliterated according to: "B" stands for ( ) " $\beta$ "; "G" " $\gamma$ "; "D" " $\delta$ "; " $E$ " " $\epsilon$ "; "Z" " $\zeta$ "; and " $H$ " " $\eta$ ". 
et al., 2011), as shown in Figure 6C. For the optimal conformation, $\mathrm{HgCl}_{2}$ is surrounded by amino acid residues (His 94, His 296, and Glu 98), whilst the suboptimal conformation is located adjacent to amino acid residues (His 263, Ala 286, and Val 283). The detailed information of interaction between the amino acid residues and $\mathrm{HgCl}_{2}$ is summarized in Table $\mathbf{1}$. As we all know, the catalytic center of tyrosinase is like a pocket, which consists of two $\mathrm{Cu}$ ions and six histidine residues, including His 61, His 85, His 94, His 259, His 263, and His 296 (Ismaya et al., 2011). Therefore, it can be inferred that the $\mathrm{HgCl}_{2}$ may change the morphology of catalytic center by binding with the amino acid residues, such as His 94, His 296, and His 263. Some experiments (in next section) were furtherly designed to support this point.

\section{Further Exploration of Binding Sites}

Amino acids, including His, $\mathrm{Val}$, Glu, and Ala, were checked to see whether they have any impact on the inhibitory effect of $\mathrm{HgCl}_{2}$ on tyrosinase. It was found that only His exhibited the significant effect on protecting the diphenolase activity in the presence of $\mathrm{HgCl}_{2}$, as shown in Figure 7A. We also found that His took effects in a concentration-dependent manner, and the protecting capability increased from $48.83 \pm 1.89 \%$ to $83.43 \pm 2.56 \%$ with the increasing concentrations ranged from 10.42 to $666.67 \mu \mathrm{mol} / \mathrm{L}$, which is illustrated in Figure 7B. The effects of His on monophenolase activity in the presence of $\mathrm{HgCl}_{2}$ were shown in Figure 7C, which demonstrated that His also protect the monophenolase activity when compared to Figure 1B. Val, Glu, and Ala still exhibited no effects on protecting the monophenolase activity (Data no shown).
The results demonstrated that $\mathrm{HgCl}_{2}$ preferred to bind with His rather than other amino acid residues, such as Val, Glu, and Ala. Once $\mathrm{HgCl}_{2}$ bound to free His, it was difficult to bind it to the residual His, which resulted in the protective effects. In order to further prove the interaction between His and $\mathrm{HgCl}_{2}$, the UV-Vis spectra of $\mathrm{His}, \mathrm{HgCl}_{2}$, and the mixture of both is presented in Figure 7D; this shows the significant difference between the mixture and $\mathrm{His}$ (or $\mathrm{HgCl}_{2}$ ), suggesting the formation of His$\mathrm{HgCl}_{2}$ complexes. Thus, based on this indirect and direct evidence (results of docking study), we believe that $\mathrm{HgCl}_{2}$ exhibits inhibitory effects on tyrosinase by binding to the amino residual (His) of the catalytic center of tyrosinase.

\section{CONCLUSIONS}

In this paper, kinetics studies, different spectroscopic measurements, and molecular docking studies were employed to explore the inhibitory effects and mechanism of $\mathrm{HgCl}_{2}$ on tyrosinase. For monophenolase, $\mathrm{HgCl}_{2}$ could obviously prolong its lag time and decrease its steady-state rate with the $\mathrm{IC}_{50}$ value of $29.97 \mu \mathrm{mol} / \mathrm{L}$. A kinetic study showed that $\mathrm{HgCl}_{2}$ could also significantly inhibit the diphenolase activity with the $\mathrm{IC}_{50}$ value of $77.93 \mu \mathrm{mol} / \mathrm{L}$ in an irreversible non-competitive manner. $\mathrm{HgCl}_{2}$ quenched the fluorescence of tyrosinase by a static procedure and formed an irreversible complex with $K_{\mathrm{i}}$ value of $29.41 \mu \mathrm{mol} / \mathrm{L}$. The molecular docking study suggested that $\mathrm{HgCl}_{2}$ bound to the His residual of the catalytic center of tyrosinase and might change the morphology, leading to the inhibitory effects. Our studies firstly demonstrated that
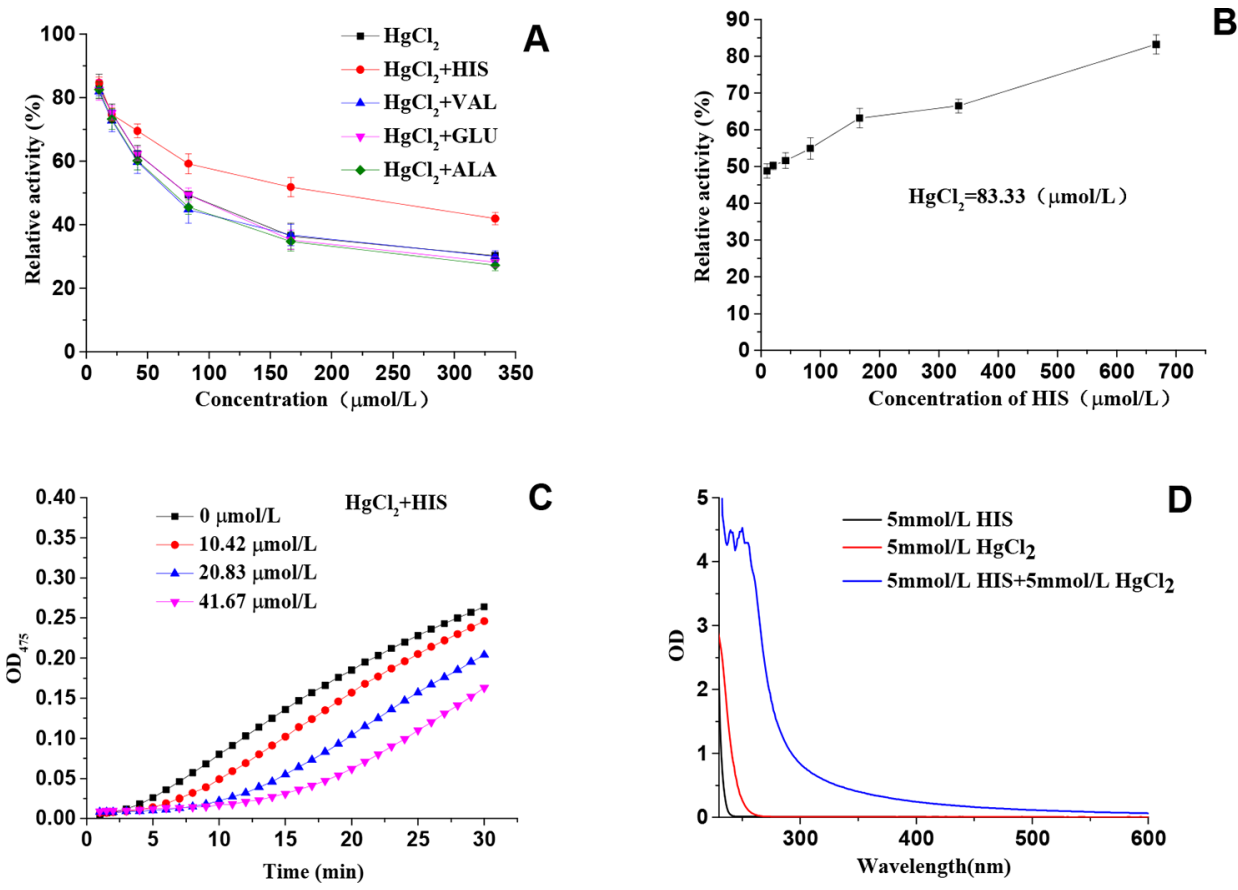

FIGURE 7 | (A) Effects of amino acids on protecting the diphenolase activity in the presence of $\mathrm{HgCl}_{2}$; (B) The relationship between the protecting effects and the concentration of His; (C) Effects of His on protecting the monophenolase activity in the presence of $\mathrm{HgCl}_{2}$; (D) The UV-Vis spectra of $\mathrm{His}, \mathrm{HgCl}_{2}$, and a mixture of both. The experiment was repeated three times $(n=3)$. 
$\mathrm{HgCl}_{2}$ has the capability to inhibit tyrosinase directly, which is helpful to further our understanding of the inhibition mechanism of mercury on tyrosinase and to expand the scientific understanding of the toxicity of mercury in organisms.

\section{DATA AVAILABILITY STATEMENT}

The datasets generated for this study are available on request to the corresponding author.

\section{AUTHOR CONTRIBUTIONS}

JC, ZR, and NJ contributed conception and design of the study and performed the docking study. YY, MR, and QL designed and

\section{REFERENCES}

Abouzied, O. K., and Alshihi, O. I. K. (2008). Characterization of subdomain IIA binding site of human serum albumin in its native, unfolded, and refolded states using small molecular probes. J. Am. Chem. Soc. 130, 10793-10801. doi: $10.1021 /$ ja8031289

Agrawal, S. S., and Mazhar, M. (2015). Adulteration of mercury in skin whitening creams - a nephrotoxic agent. Curr. Med. Res. Pract. 5, 172-175. doi: 10.1016/ j.cmrp.2015.07.007

Al-Saleh, I. (2016). Potential health consequences of applying mercury-containing skin-lightening creams during pregnancy and lactation periods. Int. J. Hyg. Environ. Health 219, 468-474. doi: 10.1016/j.ijheh.2016.03.002

Barry, L., Nisha, M., and Kundu, R. V. (2011). Widespread use of toxic skin lightening compounds: medical and psychosocial aspects. Dermatol. Clinics 29, 111-123. doi: 10.1016/j.det.2010.08.010

Boyd, A. S., Seger, D., Vannucci, S., Langley, M., Abraham, J. L., and King, L. E. (2000). Mercury exposure and cutaneous disease. J. Am. Acad. Dermatol. 43, 81-90. doi: $10.1067 / \mathrm{mjd} .2000 .106360$

Chan, T. Y. K. (2011). Inorganic mercury poisoning associated with skinlightening cosmetic products. Clin. Toxicol. 49, 886-891. doi: 10.3109/ 15563650.2011.626425

Chen, Q.-X., Song, K.-K., Qiu, L., Liu, X.-D., Huang, H., and Guo, H.-Y. (2005). Inhibitory effects on mushroom tyrosinase by p-alkoxybenzoic acids. Food Chem. 91, 269-274. doi: 10.1016/j.foodchem.2004.01.078

Chen, J., Yu, X., and Huang, Y. (2016). Inhibitory mechanisms of glabridin on tyrosinase. Spectrochim Acta A Mol. Biomol. Spectrosc 168, 111-117. doi: 10.1016/j.saa.2016.06.008

Chen, J., Liu, S., Huang, Z., Huang, W., Li, Q., and Ye, Y. (2017). Molecular inhibitory mechanism of dihydromyricetin on mushroom tyrosinase. J. Biomol. Struct. Dyn. 36, 3740-3752. doi: 10.1080/07391102.2017.1397059

Clarkson, T. W., Magos, L., and Myers, G. J. (2003). The toxicology of mercury current exposures and clinical manifestations. New Engl. J. Med. 349, 17311737. doi: 10.1056/NEJMra022471

Donghyun, K., Jiyeoun, P., Jinhee, K., Cheolkyu, H., Jeonghyeok, Y., Namdoo, K., et al. (2006). Flavonoids as mushroom tyrosinase inhibitors: a fluorescence quenching study. J. Agric. Food Chem. 54, 935-941. doi: 10.1021/jf0521855

Fan, M., Zhang, G., Hu, X., Xu, X., and Gong, D. (2017). Quercetin as a tyrosinase inhibitor: inhibitory activity, conformational change and mechanism. Food Res. Int. 100, 226-233. doi: 10.1016/j.foodres.2017.07.010

Germanò, M. P., Cacciola, F., Donato, P., Dugo, P., Certo, G., D'angelo, V., et al. (2012). Betula pendula leaves: polyphenolic characterization and potential innovative use in skin whitening products. Fitoterapia 83, 877-882. doi: 10.1016/j.fitote.2012.03.021

Hamann, C. R., Boonchai, W., Wen, L., Sakanashi, E. N., Chu, C.-Y., Hamann, K., et al. (2014). Spectrometric analysis of mercury content in 549 skin-lightening products: is mercury toxicity a?hidden?global health hazard? J. Am. Acad. Dermatol. 70, 281-287.e283. doi: 10.1016/j.jaad.2013.09.050 performed the inhibitory effects of $\mathrm{HgCl}_{2}$ on tyrosinase. All authors contributed to manuscript revisions and read and approved the submitted version.

\section{FUNDING}

This work was supported by the Natural Science Foundation of Fujian Province (grant number 2019J01806), the Education Department of Fujian Province (grant number JZ160470), the Putian Science and Technology Bureau (grant number 2018SP3004), and the Training Program of Innovation and Entrepreneurship for Undergraduates (grant number 201811498003, 201811498027, and 201911498055).

Hridya, H., Amrita, A., Mohan, S., Gopalakrishnan, M., Dakshinamurthy, T. K. Doss, G. P., et al. (2016). Functionality study of santalin as tyrosinase inhibitor: a potential depigmentation agent. Int. J. Biol. Macromol. 86, 383-389. doi: 10.1016/j.ijbiomac.2016.01.098

Ismaya, W. T., Rozeboom, H. J., Weijn, A., Mes, J. J., Fusetti, F., Wichers, H. J., et al. (2011). Crystal structure of Agaricus bisporus mushroom tyrosinase: identity of the tetramer subunits and interaction with tropolone. Biochemistry 50, 5477-5486. doi: 10.1021/bi200395t

Kibukamusoke, J. W., Davies, D. R., and Hutt, M. S. (1974). Membranous nephropathy due to skin-lightening cream. Br. Med. J. 2, 646-647. doi: 10.1136/bmj.2.5920.646

Körner, A. M., and Pawelek, J. M. (1982). Mammalian tyrosinase catalyzes three reactions in the biosynthesis of melanin. Science 217, 1163-1165. doi: 10.1126/ science. 6810464

Lerner, A. B. (1952a). Effect of ions on melanin formation. J. Invest. Dermatol. 18, 47-52. doi: 10.1038/jid.1952.6

Lerner, A. B. (1952b). Mammalian tyrosinase: effect of ions on enzyme action. Arch. Biochem. Biophys. 36, 473-481. doi: 10.1016/0003-9861(52)90435-9

Lin, M. Z., Chai, W. M., Chong, O. Y., Huang, Q., Xu, X. H., and Peng, Y. Y. (2018). Antityrosinase mechanism of omeprazole and its application on the preservation of fresh-cut Fuji apple. Int. J. Biol. Macromol. 117, 538-545. doi: 10.1016/j.ijbiomac.2018.05.172

Morris, G. M., Huey, R., and Olson, A. J. (2008). Using AutoDock for ligandreceptor docking. Curr. Protoc. Bioinf. Chapter 8, Unit 8.14. 24 (1), 1-40. doi: 10.1002/0471250953.bi0814s24

$\mathrm{Mu}, \mathrm{Y}$., Li, L., and Hu, S.-Q. (2013). Molecular inhibitory mechanism of tricin on tyrosinase. Spectrochim. Acta A Mol. Biomol. Spectrosc. 107, 235-240. doi: 10.1016/j.saa.2013.01.058

Olumide, Y. M., Akinkugbe, A. O., Dan, A., Tahir, M., Ngozi, A., Shola, A., et al. (2008). Complications of chronic use of skin lightening cosmetics. Int. J. Dermatol. 47, 344-353. doi: 10.1111/j.1365-4632.2008.02719.x

Park, K., Park, Y., Lee, J., Hahn, H., Lee, S., Bae, C., et al. (2005). Inhibition kinetics of mushroom tyrosinase by copper-chelating ammonium tetrathiomolybdate. Biochim. Et Biophys. Acta Gen. Subj. 1726, 115-120. doi: 10.1016/ j.bbagen.2005.06.010

Qiu, L., Chen, Q. Q., Huang, H., and Song, K. K. (2005). Irreversibly inhibitory kinetics of 3,5-dihydroxyphenyl decanoate on mushroom (Agaricus bisporus) tyrosinase. Bioorganic Med. Chem. 13, 6206-6211. doi: 10.1016/j.bmc.2005.06.034

Seo, S. Y., And, V. K. S., and Sharma, N. (2003). Mushroom Tyrosinase: Recent Prospects. J. Agric. Food Chem. 51, 2837-2853. doi: 10.1021/jf020826f

Soares, M. A., Almeida, M. A., Marins-Goulart, C., Chaves, O. A., Echevarria, A., and Mcc, D. O. (2017). Thiosemicarbazones as inhibitors of tyrosinase enzyme. Bioorganic Med. Chem. Lett. 27, 3546-3550. doi: 10.1016/j.bmcl.2017.05.057

Tan, X., Song, Y. H., Park, C., Lee, K. W., Kim, J. Y., Kim, D. W., et al. (2016). Highly potent tyrosinase inhibitor, neorauflavane from Campylotropis hirtella and inhibitory mechanism with molecular docking. Bioorganic Med. Chem. 24, 153-159. doi: 10.1016/j.bmc.2015.11.040 
Wang, Y., Zhang, G., Yan, J., and Gong, D. (2014). Inhibitory effect of morin on tyrosinase: insights from spectroscopic and molecular docking studies. Food Chem. 163, 226-233. doi: 10.1016/j.foodchem.2014.04.106

Wang, Y., Zhang, G., Pan, J., and Gong, D. (2015). Novel insights into the inhibitory mechanism of kaempferol on xanthine oxidase. J. Agric. Food Chem. 63, 526-534. doi: 10.1021/jf505584m

Yoon, J., Fujii, S., and Solomon, E. I. (2009). Geometric and electronic structure differences between the type 3 copper sites of the multicopper oxidases and hemocyanin/tyrosinase. Proc. Natl. Acad. Sci. U. S. A. 106, 6585-6590. doi: 10.1073/pnas.0902127106
Conflict of Interest: The authors declare that the research was conducted in the absence of any commercial or financial relationships that could be construed as a potential conflict of interest.

Copyright (C) 2020 Chen, Ye, Ran, Li, Ruan and Jin. This is an open-access article distributed under the terms of the Creative Commons Attribution License (CC BY). The use, distribution or reproduction in other forums is permitted, provided the original author(s) and the copyright owner(s) are credited and that the original publication in this journal is cited, in accordance with accepted academic practice. No use, distribution or reproduction is permitted which does not comply with these terms. 